\title{
Unsteady Stagnation Point Flow of Nanofluid over a Stretching/Shrinking Sheet in a Porous Medium
}

\author{
Ali Shomali ${ }^{1}$, Abbas Panahi ${ }^{2,3}$, Mohammad Hossein Sabour ${ }^{2}$, Meysam Mosania $^{2}$ \\ ${ }^{1}$ School of Mechanical Engineering, University of Tehran \\ Tehran, Iran \\ Ali.shomali@ut.ac.ir \\ ${ }^{2}$ Department of Aerospace Engineering, FNST, University of Tehran \\ Tehran, Iran \\ sabourmh@ut.ac.ir; meysam.moosania@ut.ac.ir \\ ${ }^{3}$ SHEZAN Research \& Innovation Centre, Pardis Technology Park \\ Tehran, 1657167333, Iran \\ abbas.panahi@ut.ac.ir
}

\begin{abstract}
In this paper two dimensional stagnation point of unsteady nanofluid flow over a stretching/shrinking sheet embedded in a porous medium is analyzed numerically and effects of porosity, heat generation and volume fraction on Nusselt number, skin friction coefficient and convergence time and velocity and temperature distribution are investigated in detail. Also different behaviors of stretching and shrinking sheets are observed. Results are reported for three different volume fraction values $(0-0.1-0.2)$. It is found that velocity step change increases skin friction coefficient while it has opposite effect on Nusselt number. Stretching sheet showed higher variation in skin friction coefficient value with time while shrinking sheet presented higher variation of Nusselt number.
\end{abstract}

Keywords: Unsteady Flow, Stagnation Point, Nanofluid, Numerical Solution, Porous Medium, Heat Generation.

\section{Introduction}

In recent years Nanofluids have found a major interest in both industry and research. Every Nanofluid consists of a base fluid such as water or ethylene glycol and ultra-fine solid particles (1-100nm) called Nano particles which are suspended in the base fluid [1]. These Nano particles can be both metallic and nonmetallic, these additives are used to enhance transport properties and heat transfer characteristics of base fluid. Choi [2] used the term "Nanofluid" for the mixture of Nano particles and base fluid for the first time. Nanofluids have several applications such as in power generation, automotive, aerospace, biomechanics, etc. also Nanofluids are used as coolants for engines and electronic devices. Saidur et al. [3] studied applications and further challenges in usage of Nanofluids. Also Wongwises and Trisaksri [4] focused on different Nanofluids and their characteristics compared to each other in their study and analyzed their behavior in, forced and natural convection and boiling heat transfer.

Studying Heat transfer coefficient in natural and forced convection problems with different flow regimes and comparing their abilities over basic fluid for investigating Nanofluid advantages has found an increasing interest in recent years. Masuda et al. [5] investigated alternation of nanofluids thermal conductivity by dispersing small amount of ultraFine particles of $\mathrm{Al}_{2} \mathrm{O}_{3}, \mathrm{SiO}_{2}$ and $\mathrm{TiO}_{2}$ into it. Studies on Nanofluid thermal conductivity resulted that base fluid and Nanoparticles thermal conductivity, volume fraction, Nano particles size and shape and volume to surface ratio are important factors that influence conductive heat transfer of Nanofluids [4], Hwang et al. [6] suggested that nanofluids thermal conductivity is influenced mostly by base fluid and Nanoparticles thermal conductivity. Also, Yoo et al. [7] found the volume to surface ratio as important factor that effects nanofluid thermal conductivity. Most of experimental and numerical studies show enhancement in thermal conductivity by adding Nano particles to base fluid, for example Choi [1] reported high thermal conductivity enhancement by using copper Nano particles in water and also he reported $150 \%$ increase in poly ( $\alpha$-olefin) oil thermal conductivity by adding Nano particles at $1 \%$ volume fraction.

Many theoretical and experimental studies have been performed on nanofluid convective heat transfer, for example Daungthongsukand and Wongwises [8] and Kakaç and Pramuanjaroenkij [9] summarized important researches on 
convective heat transfer of nanofluids. Also, Ding and Wen [10] research are examples of experimental studies on Convective heat transfer. Unlike thermal conduction and forced convection, experiments on natural convection show reduction in heat transfer by utilizing nanofluids. Ding and Wen [11] discussed possible reasons for this behavior.

Boundary layer flow and heat transfer of nanofluids on stretching/shrinking sheets embedded in porous medium has attracted a great deal of attention in recent years because of its broad application in metallurgy and mechanical forming processes, in these processes quality of the final product is a function of skin fraction coefficient and heat transfer rate [12]. Lakshmisha et al. [13] performed a numerical study on heat and mass transfer of Nanofluids on 3-D unsteady flow over a stretching sheet. Backok et al. [14] transformed partial deferential equations to ordinary ones and studied heat transfer of unsteady boundary layer flow on stretching/shrinking sheet and found that dual solutions exist for both stretching and shrinking cases. Also, Backok [15] analyzed nanofluids flow and its heat transfer on a porous rotational plane. Elbashbeshy and Bazid [16] carried out a similar study with heat source and isothermal condition for the plane and studied effects of prandtl number, permeability, suction and heat generation parameter on velocity, temperature and thermal boundary layer thickness, they also concluded that heat transfer rate decreases with heat generation and permeability and increases with prandtl number. Nazer et al. [17] analyzed unsteady boundary layer near stagnation point for stretching sheet. Hamad [18] studied stagnation point flow on stretching/shrinking sheet for 3 different nanofluids and analyzed the effect of various parameters such as nanofluid type and volume fraction on heat transfer and friction coefficient, also Rana [19] studied flow on stretching sheet with nonlinear velocity. Pal and Mandel [20] research is One of the recent numerical studies on mixed convection steady stagnation point flow on stretching/shrinking sheet problem which considered the effect of permeability, heat generation, radiation and suction/injection in their solution. Numerous studies have been performed to analyze magnetic field effect on flow and heat transfer. Elbashbeshy [21] studied influence of radiation and magnetic fluid on unsteady flow on stretching sheet. One of the basic assumptions in nanofluids problems is no slip flow which assumes that no slip occurs between base fluid and nanoparticles, Van gorder [22] discussed slip flow on their studies.

Objective of this paper is to study unsteady stagnation point flow with mixed convection heat transfer on porous stretching/shrinking sheet in the presence of heat generation and radiation when a sudden-step change happens in plane velocity by using similarity solutions. Skin-friction coefficient, local Nusselt number and convergence time are reported and examined. Particles and base fluid are assumed in thermal equilibrium and there is no-slip between base fluid and nanoparticles.

\section{Formulation of the Problem}

We consider an unsteady two dimensional stagnation point laminar flow of a viscous incompressible nanofluid over a permeable stretching/shrinking sheet with heat generation/absorption surrounded by a porous media. $\mathrm{y}$ is the coordinate normal to sheet surface and $\mathrm{x}$ is the coordinate along the sheet It's assumed that velocity of free stream is $U_{x}=a x$ where ' $\mathrm{a}$ ' is a constant, $v_{w}(x, t)$ is mass flux velocity from sheet with $v_{w}(x, t)>0$ for injection and $v_{w}(x, t)<0$ for suction. Also it's Assumed that Wall is stretched or shrunk with the velocity $u_{w}= \pm c x$ (c is a constant) when a step change happens in its velocity by change in its constant $c^{*}= \pm 0.1 c$, so that changed velocity becomes $u^{*}= \pm\left(c+c^{*}\right) x$. Also we have $T_{\infty}$ for constant nanofluid ambient temperature and $T_{w}$ for constant wall temperature, $Q_{0}$ is heat generation/absorption parameter $\left(Q_{0}>0\right.$ for heat generation and $Q_{0}<0$ for heat absorption), also " $\mathrm{t}$ " represents time and $\mathrm{u}$ and $\mathrm{v}$ are velocities along $\mathrm{x}$ and $\mathrm{y}$ axis. Schematic diagram of the problem, coordinate system and physical model is shown in figure 1.

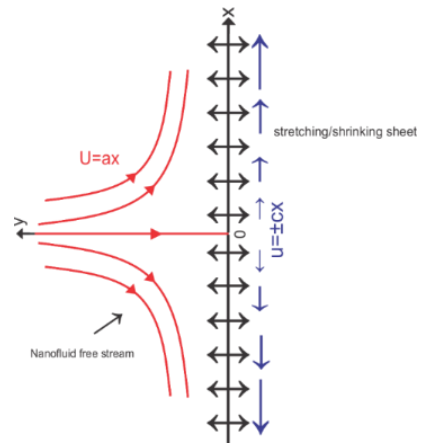

Fig. 1: Schematic diagram of the problem. 
Incompressibility, Thermal equilibrium and no slip condition are some of basic assumptions in this study which we already discussed. Under these assumptions continuity and unsteady boundary layer equations of momentum and energy for an incompressible and viscous nanofluid with considering thermal radiation, heat source or sink and mass injection or suction are:

$$
\begin{gathered}
\frac{\partial u}{\partial x}+\frac{\partial v}{\partial y}=0 \\
\frac{\partial u}{\partial t}+u \frac{\partial u}{\partial x}+v \frac{\partial u}{\partial y}=U_{(x)} \frac{d U_{(x)}}{d x}+\frac{\mu_{n f}}{\rho_{n f}} \frac{\partial^{2} u}{\partial y^{2}}+\frac{\mu_{n f}}{\rho_{n f} K}\left(U_{(x)}-u\right)+\frac{(\rho \beta)_{n f}}{\rho_{n f}} g\left(T-T_{\infty}\right) \\
\frac{\partial T}{\partial t}+u \frac{\partial T}{\partial x}+v \frac{\partial T}{\partial y}=\alpha_{n f} \frac{\partial^{2} T}{\partial y^{2}}-\frac{1}{\left(\rho C_{p}\right)_{n f}} \frac{\partial q_{r}}{\partial y}+\frac{Q_{0}}{\left(\rho C_{p}\right)_{n f}} g\left(T-T_{\infty}\right)
\end{gathered}
$$

Boundary conditions considered for stretching/shrinking sheet in initial case $(\mathrm{t}=0)$ are:

$$
\begin{array}{ll}
u=u_{w}= \pm c x v=v_{w} T=T_{w}=T_{\infty}+b(x / l) & \text { at } y=0 \\
u \rightarrow U_{(x)}=a x v \rightarrow 0 T \rightarrow T_{w} & \text { as } y \rightarrow \infty
\end{array}
$$

And Boundary conditions considered for stretching/shrinking sheet after step change case $(t>0)$ are:

$$
u=u_{w}(x)=(c \pm 0.1 c) x=c^{*} x \quad v=v_{w} T_{w}=T_{\infty}+\frac{b}{l} x \quad \text { at } \quad y=0
$$

$\mathrm{T}$ is the temperature of nanofluid, $\rho_{n f}$ is nanofluid density, $\kappa_{n f}$ is thermal conductivity of nanofluid $\mu_{n f}$ is nanofluid the coefficient of viscosity, $\alpha_{n f}$ is thermal diffusivity of nanofluid, $\beta_{n f}$ is nanofluid thermal expansion and $\left(\rho C_{P}\right)_{n f}$ is nanofluid heat capacity. These thermophysical parameters are defined as follow [25]:

$$
\begin{gathered}
\alpha_{n f}=\frac{k_{n f}}{\left(\rho C_{p}\right)_{n f}} \quad \rho_{n f}=(1-\phi) \rho_{f}+\phi \rho_{s} \\
(\rho \beta)_{n f}=(1-\phi)(\rho \beta)_{f}+\phi(\rho \beta)_{s} \\
\left(\rho C_{p}\right)_{n f}=(1-\phi)\left(\rho C_{p}\right)_{f}+\phi\left(\rho C_{p}\right)_{s} \\
\frac{k_{n f}}{k_{f}}=\frac{\left(k_{s}+2 k_{f}\right)-2 \phi\left(k_{f}-k_{s}\right)}{\left(k_{s}+2 k_{f}\right)+2 \phi\left(k_{f}-k_{s}\right)}
\end{gathered}
$$

And nanofluid viscosity as suggested by Brinkman [32]:

$$
\frac{\mu_{n f}}{\mu_{f}}=\frac{1}{(1-\phi)^{2.5}}
$$

$\phi$ Is volume fraction of Nanoparticles in base fluid, $\rho_{f}$ is base fluid density, $\rho_{s}$ is nanoparticle density, $\mu_{f}$ is viscosity of base fluid, $\kappa_{f}$ is base fluid thermal conductivity, ${ }_{s}$ represents nanoparticles thermal conductivity, $\left(\rho C_{P}\right)_{f}$ is base fluid heat capacity and $\left(\rho C_{P}\right)_{s}$ represents nanoparticle heat capacity. Radiation heat flux is formulated by 
considering Rosseland approximation. Pal and Mandal [20] derived this equation by expanding $T^{4}$ as Taylor series near $^{T_{\infty}}$ :

$$
\frac{\partial q_{r}}{\partial y}=-\frac{16 \sigma^{*} T_{\infty}^{3}}{3 K^{*}} \frac{\partial^{2} T}{\partial y^{2}}
$$

We use non-dimensional variables and introduce the following non-dimensional time variable:

$$
\psi=\sqrt{c \vartheta_{f}} x F_{(\eta)}, \quad \theta_{(\eta)}=\frac{T-T_{\infty}}{T_{w}-T_{\infty}}, \quad \eta=\sqrt{\frac{c}{\vartheta_{f}}} y, t^{*}=t c
$$

$\psi$ Is stream function, thus continuity equation (1) is satisfied identically. With these new similarly variables, velocities in $\mathrm{x}$ and $\mathrm{y}$ directions are:

$$
u=\frac{\partial \psi}{\partial y}=c x F_{(\eta)}^{\prime} v=-\frac{\partial \psi}{\partial x}=\sqrt{c \vartheta_{f}} F_{(\eta)}
$$

Substituting equations (9) and (10) in equation (2) momentum equation (10) becomes:

$$
\begin{gathered}
F_{t^{*}}^{\prime}=F F^{\prime \prime}-{F^{\prime}}^{2}+\frac{a^{2}}{c^{2}}+\mathrm{A}\left(F^{\prime \prime \prime}+K_{1}\left(\frac{a}{c}-F^{\prime}\right)\right)+\mathrm{B} \Lambda \theta \\
\mathrm{A}=\frac{1}{(1-\phi)^{2.5}\left(1-\phi+\phi \frac{\rho_{s}}{\rho_{f}}\right)} \\
\mathrm{B}=\frac{\left(1-\phi+\phi \frac{(\rho \beta)_{s}}{(\rho \beta)_{f}}\right)}{\left(1-\phi+\phi \frac{\rho_{s}}{\rho_{f}}\right)}
\end{gathered}
$$

Substituting equations (9) and (10) in equation (3), energy equation (11) becomes:

$$
\begin{gathered}
\theta_{\xi}=\frac{\Gamma}{P r_{e f f}} \theta^{\prime \prime}+\left(F \theta^{\prime}-F^{\prime} \theta\right)+\Gamma \lambda \theta \\
\Gamma=\frac{\left(\rho C_{p}\right)_{f}}{\left(\rho C_{p}\right)_{n f}}
\end{gathered}
$$

Boundary conditions for unsteady problem are:

$$
\begin{array}{ccccc}
F=S, & F^{\prime}=1 \pm 0.1 \quad \theta=1 & \text { at } & \eta=0 & \text { (for stretching sheet) } \\
F=S, & F^{\prime}=-1 \pm 0.1 \quad \theta=1 & \text { at } & \eta=0 & \text { (for shrinking sheet) } \\
& F^{\prime} \rightarrow \frac{a}{c} \theta \rightarrow 0 & & \text { as } & \eta \rightarrow \infty
\end{array}
$$


Where $F_{\xi}^{\prime}$ and $\theta_{\xi}$ are $F^{\prime}$ and $\theta$ time derivatives respectively, $\lambda=\frac{Q_{0}}{\left(\rho C_{p}\right)_{f}}$ is heat source/sink parameter, $S=-\frac{v_{w}}{\sqrt{C v_{f}}}$ is mass flux parameter $K=\frac{\vartheta_{f}}{k C}$ is porous parameter $\Lambda=\frac{g(\rho \beta)_{f} b}{\rho_{f} C^{2} l}$ is mixed convection parameter, $P r_{e f f}=\frac{P r}{\frac{k_{n f}}{k_{f}}+N_{r}}$ is effective Prandtl number where $P r=\frac{v_{f}}{\alpha_{f}}$ is Prandtl number and $N_{r}=-\frac{16 \sigma^{*} T_{\infty}^{3}}{3 K^{*} k_{f}}$ is radiation parameter.

Pal and Mandal [27] used these symbols for making their basic equations simpler to study and showing their nondimensional effective parameters on solution. None dimensionalizing of equations is done by initial velocity constant 'c' so after step change in velocity our differential equations don't change and only boundary conditions change.

It is assumed that the flow is steady at time $t^{*}=0$ and becomes unsteady for $t^{*}>0$ Due to a step change in the velocity of the sheet. Hence the initial condition are given by the steady-state equations obtained by substituting $F_{t^{*}}^{\prime}=$ $\theta_{t^{*}}=0$ in equations (12) and (13):

$$
\begin{gathered}
F F^{\prime \prime}-{F^{\prime}}^{2}+\frac{a^{2}}{c^{2}}+\mathrm{A}\left(F^{\prime \prime \prime}+K_{1}\left(\frac{a}{c}-F^{\prime}\right)\right)+\mathrm{B} \Lambda \theta=0 \\
\frac{\Gamma}{P r_{e f f}} \theta^{\prime \prime}+\left(F \theta^{\prime}-F^{\prime} \theta\right)+\Gamma \lambda \theta=0
\end{gathered}
$$

The corresponding boundary condition are

$$
\begin{aligned}
& F=S \quad F^{\prime}=1 \quad \theta=1 \quad \text { at } \quad \eta=0 \quad \text { (for stretching sheet) } \\
& F=S \quad F^{\prime}=-1 \quad \theta=1 \text { at } \eta=0 \text { (for shrinking sheet) } \\
& F^{\prime} \rightarrow \frac{a}{c} \theta \rightarrow 0 \quad \text { as } \quad \eta \rightarrow \infty
\end{aligned}
$$

Also Boundary conditions as as $y \rightarrow \infty$ remain unchanged. Skin friction coefficient and local Nusselt number are defined by:

$$
\begin{array}{cl}
C_{f}=\frac{2 \mu_{n f}}{\rho_{f} u_{w}^{2}}\left(\frac{\partial u}{\partial y}\right)_{y=0} & \text { (skin-friction coefficient) } \\
N u_{x}=\frac{-x k_{n f}}{k_{f}\left(T_{w}-T_{\infty}\right)}\left(-\frac{\partial T}{\partial y}\right)_{y=0} \quad \text { (local Nusselt number) }
\end{array}
$$

Substituting equations (17) and (18) in equations (13) and (14) .Skin friction coefficient and local Nusselt number are derived as:

$$
R e_{x}^{1 / 2} C_{f}=\frac{2}{(1-\phi)^{2.5}} F^{\prime \prime}(0) \quad R e_{x}^{1 / 2} N u_{x}=-\frac{k_{n f}}{k_{f}} \theta^{\prime}(0)
$$

\section{Results and Discussion}

By using similarity variables (9), nonlinear partial differential equations of momentum (2) and energy (3) are transformed into coupled linear third-order deferential equations (10) and (11) which are easier to be studied and solved numerically. A numerical code based on the finite-difference method has been developed to solve nonlinear

Differential equations (10) and (11) subject to boundary conditions (13) and initial condition (14) and (15). Numerical method chosen for this study is Forth order Runge-Kutta method which its accuracy for our problem is validated by previous studies on similar steady problem [27]. 
In order to analyze the effect of permeability parameter, heat generation and volume fraction in unsteady problem, numerical results are presented in figures 5-8. Table-1 shows thermophysical properties for base fluid and Nanoparticles used for this study.

Table 1: thermophysical properties of base fluid and Nano particle materials.

\begin{tabular}{|cllll|}
\hline Physical properties & $\begin{array}{l}\text { Base fluid } \\
\text { (water) }\end{array}$ & \multicolumn{1}{c}{$\mathrm{Cu}$} & $\mathrm{Al}_{2} \mathrm{O}_{3}$ & $\mathrm{TiO}_{2}$ \\
\hline $\mathrm{C}_{\mathrm{p}}(\mathrm{J} / \mathrm{kg} . \mathrm{K})$ & 4179 & 385 & 765 & 686.2 \\
$\rho\left(\mathrm{kg} / \mathrm{m}^{3}\right)$ & 997.1 & 8933 & 3970 & 4250 \\
$\mathrm{k}(\mathrm{W} / \mathrm{m} . \mathrm{K})$ & 0.613 & 400 & 40 & 8.9538 \\
$\alpha \times 10^{-7}\left(\mathrm{~m}^{2} / \mathrm{s}\right)$ & 1.47 & 1163.1 & 131.7 & 30.7 \\
\hline
\end{tabular}

In order to validate present results, data obtained from numerical method is compared with those obtained from the steady problem solved by Pal and Mandala [27]. Figure 2 shows acceptable agreement between present results and previous studies, proving that forth order Runge-Kutta method is a very accurate numerical method for this problem. It should be noted that in this numerical study, $a / c$ parameter has the constant value of 2 in order to have comparable final results. Previous studies [27] show significant change in stretching/shrinking sheet velocity and temperature distributions with different values of $a / c$.
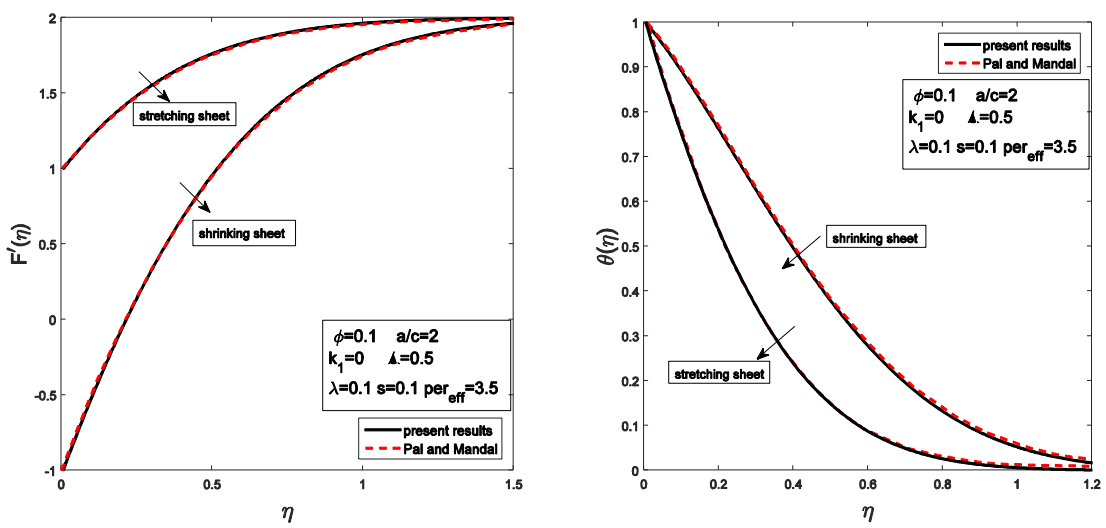

Fig. 2: the comparison of results of present study with Pal and Mandal [27].

In order to observe the variation of velocity and temperature over time, some example plots have been reported in figure 3. Figure 3 shows the initial and final temperature and velocity profile for specific values of porosity and heat generation parameters. It can be seen that stretching sheet has a higher velocity distribution than shrinking sheet whereas shrinking sheet has a higher temperature profile. This behavior can be seen for all different volume fractions and other physical parameters of the system. Unsteady velocity and temperature profiles at $c^{*}= \pm 0.1 c$ have been shown in red lines in figure 3 for a more detailed look. It can be seen that for both type of sheets, increasing sheet velocity coefficient "c" increases velocity distribution and decreases temperature distribution and decreasing this parameter "c" results opposite. It can be observed that shrinking sheet temperature profile shows higher increase/decrease with various amounts of $c^{*}$. Also shrinking sheet has a higher slope in stagnation point $(\eta=0)$ for velocity and lower slope for temperature. As expected, skin friction coefficient increases with volume fraction value. Additionally, skin friction coefficient increases for $c^{*}=-0.1 c$ and decreases for $c^{*}=+0.1 c$, this also can be seen from derivation change of velocity diagram with time near the wall in figure 3 .

Figure (4) shows the variation of stretching sheet skin-friction coefficient with time for different nanoparticle volume fractions $(\phi=0,0.1,0.2)$ at $\left(c^{*}= \pm 0.1 c\right)$ for cu-water nanofluid. It can be observed that skin friction variations for $c^{*}=0.1 c$ and $c^{*}=-0.1 c$ is different for every value of volume fraction and the differences between final steady states becomes larger by volume fraction increase. Also one can see that time needed for reaching the new 
steady state of the flow is shorter for $c^{*}=0.1 c$ compared to $c^{*}=-0.1 c$ and it decreases more by the increase of nondimensional porosity number. Skin-fraction coefficient increases with Porous parameter, this behavior was expected due to physical effects of a porous media subject to a flow. Heat generation/absorption affects momentum equation indirectly by non-dimensional temperature in buoyancy force term. Heat generation/absorption parameter increases skin friction coefficient and its growth rate is higher for larger volume fraction (due to the " $B$ " parameter that is a function of volume fraction). Figure (5) shows the variation of stretching sheet local Nusselt number with time for different nanoparticle volume fractions $(\phi=0,0.1,0.2)$ at $\left(c^{*}= \pm 0.1 c\right)$ for cu-water nanofluid. It can be seen that Energy Equation Convergence time is larger than momentum equation (roughly more than 50\%) and this condition can be seen in most of coupled momentum and energy equations due to their dependency. Convergence time is shorter for $c^{*}=+0.1 c$ compared to $c^{*}=$ $-0.1 c$ similar to momentum convergence time. Volume fraction has a considerable effect on local Nusselt number (roughly 50\% increase) and shows nanofluids potential ability for enhancing heat transfer into this group of problems. Figure 5 shows that unlike skin friction coefficient, local Nusselt number increases with $c^{*}=+0.1 c$ and decreases with $c^{*}=-0.1 c$ as could be seen previously in figure 5. Porous parameter causes a small increase in local Nusselt number and a slight decrease in convergence time also the increase in heat generation/absorption parameter causes a decrease in local Nusselt number and a small decrease in convergence time.
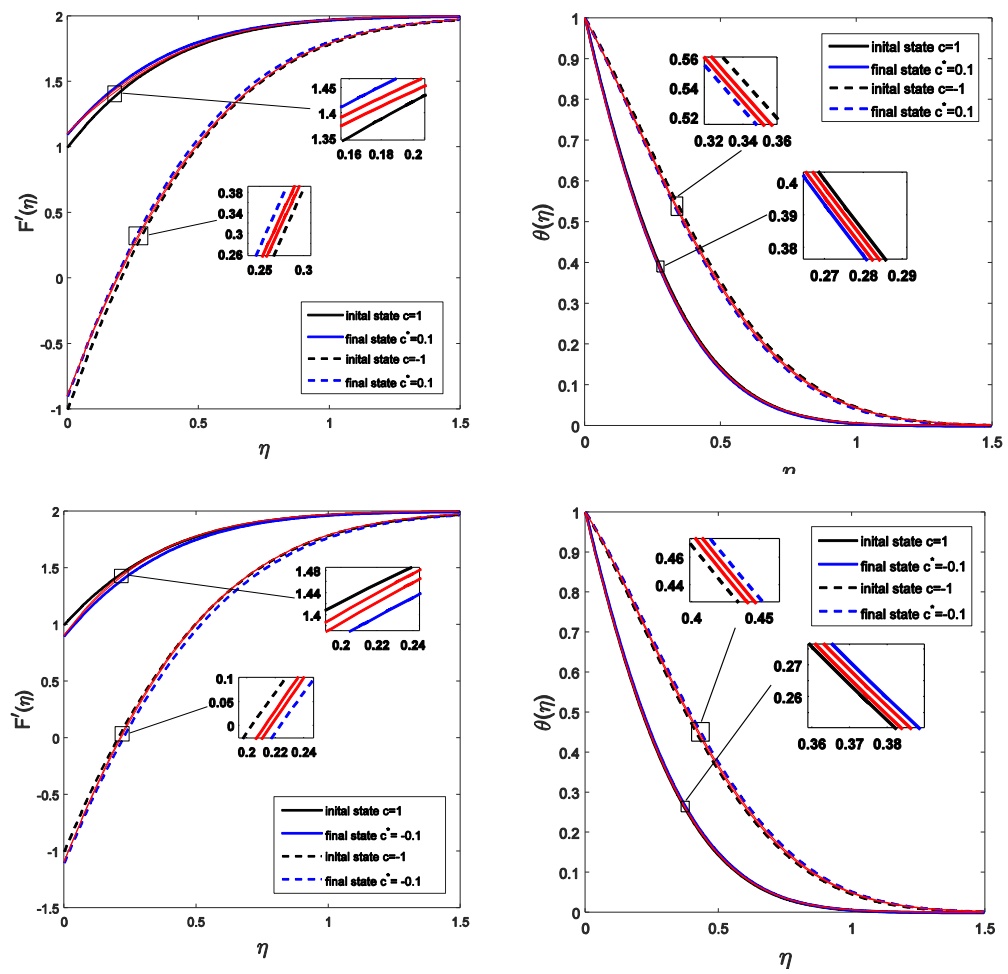

Fig. 3: initial (steady state), final and unsteady velocity and temperature profiles of stretching and shrinking sheet $\left(\operatorname{Pr}_{\text {eff }}=3.5 \mathrm{~S}=\right.$ $0.1 \phi=0.2 \lambda=0 \Lambda=0.5 \% \mathrm{a}=2 \mathrm{~K}=0)$.

Variations of skin friction coefficient and Nusselt number in shrinking sheet with time for different nanoparticle volume fractions $(\phi=0 / 0.1 / 0.2)$ at $c^{*}= \pm 0.1 c$ for cu-water nanofluid are shown respectively in figure 6 and figure 7 . These data exhibit the major difference between stretching and shrinking sheet physical behavior. Shrinking sheet convergence time is higher than stretching sheet for both momentum and energy equations. As it can be seen from figure 6, for a particular volume fraction and permeability, skin friction coefficient of shrinking sheet is always higher than stretching sheet. Heat generation parameter causes a decrease in both convergence time and Nusselt number. Although it can be observed that its affect is negligible. Similar to stretching sheet convergence time increases with volume fraction and decreases with permeability parameter. 
Unlike the skin friction coefficient, Nusselt number decreases in shrinking sheet in comparison with stretching sheet (figure 7). Convergence time decreases more with sheet speed increment due to longer time it takes for momentum equation to reach steady state. Nusselt number increases with volume fraction and permeability parameter, also heat generation causes the Nusselt number to decrease. It can be observed that convergence time increases with volume fraction and decreases with permeability and heat generation parameter.

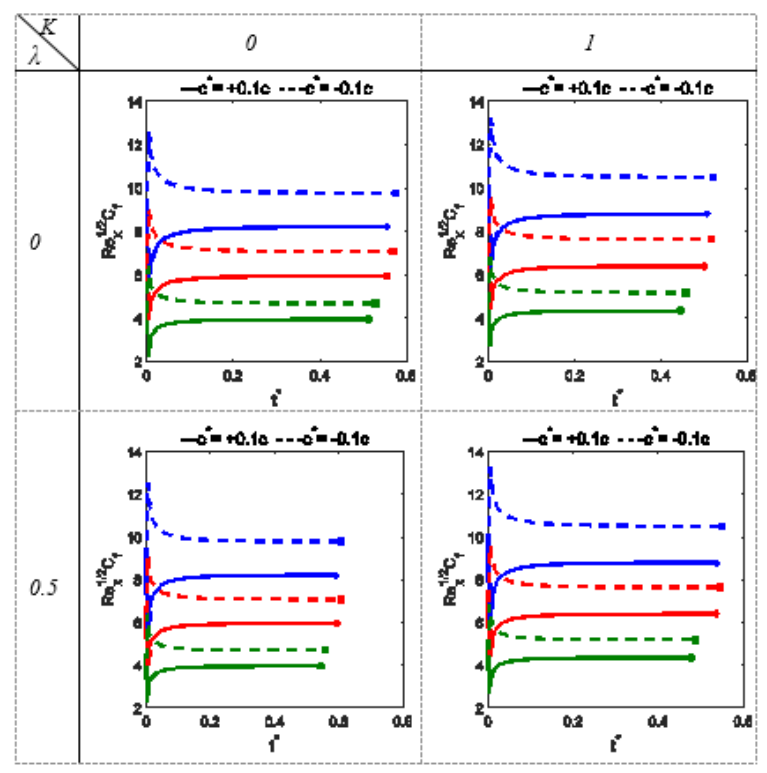

Fig. 4: variation of skin friction coefficient of stretching sheet with time for different Nano particle volume fraction $(\phi=0,0.1,0.2)$, different heat generation parameter and different porous parameter for cu-water nanofluid. $\left(\operatorname{Pr}_{\mathrm{eff}}=3.5 \mathrm{~S}=0.1 \mathrm{a} / \mathrm{c}=2 \Lambda=0.5\right)$.

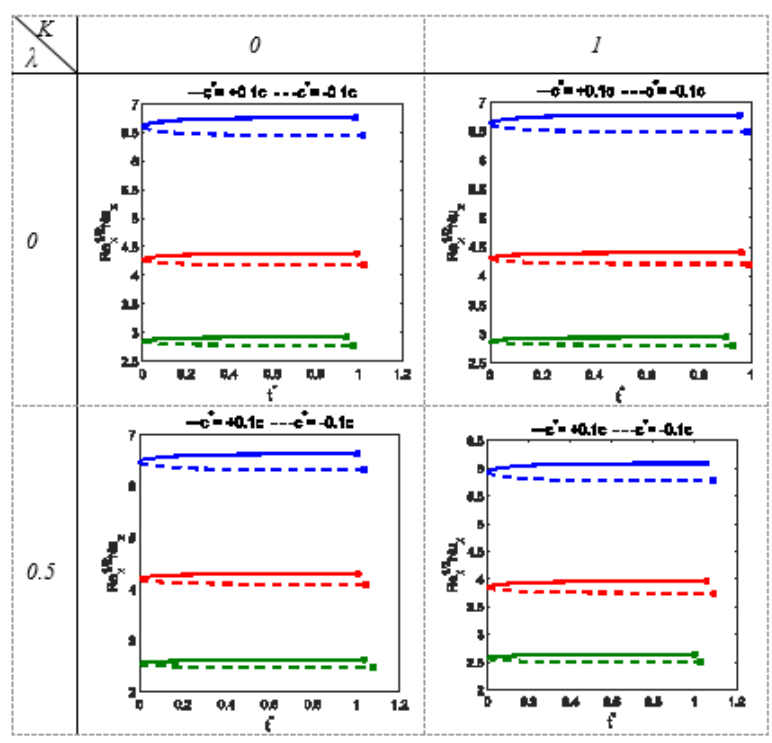

Fig. 5: variation of local Nusselt number of stretching sheet with time for different Nano particle volume fraction $(\phi=0,0.1,0.2)$, different heat generation parameter and different porous parameter for cu-water nanofluid.

$$
\left(\operatorname{Pr}_{\text {eff }}=3.5 \mathrm{~S}=0.1 \mathrm{a} / \mathrm{c}=2 \Lambda=0.5\right) \text {. }
$$




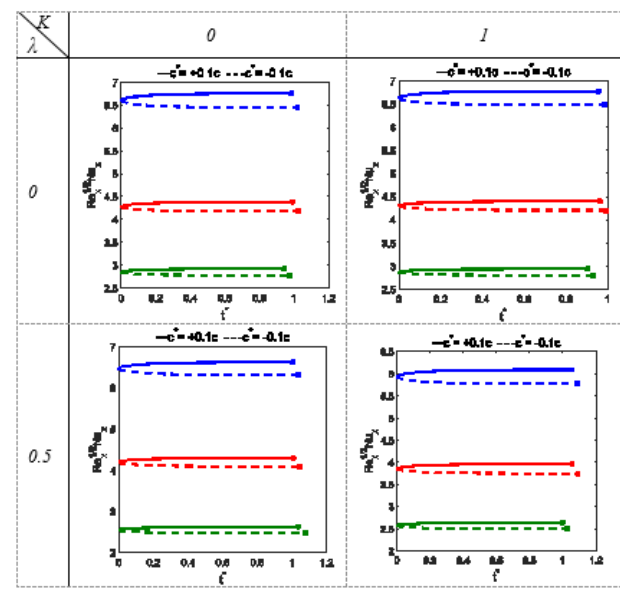

Fig. 6: variation of skin friction coefficient of shrinking sheet with time for different Nano particle volume fraction $(\phi=0,0.1,0.2)$, different heat generation parameter and different porous parameter for cu-water nanofluid. $\left(\operatorname{Pr}_{\text {eff }}=3.5 \mathrm{~S}=0.1 \mathrm{a} / \mathrm{c}=2, \Lambda=0.5\right)$

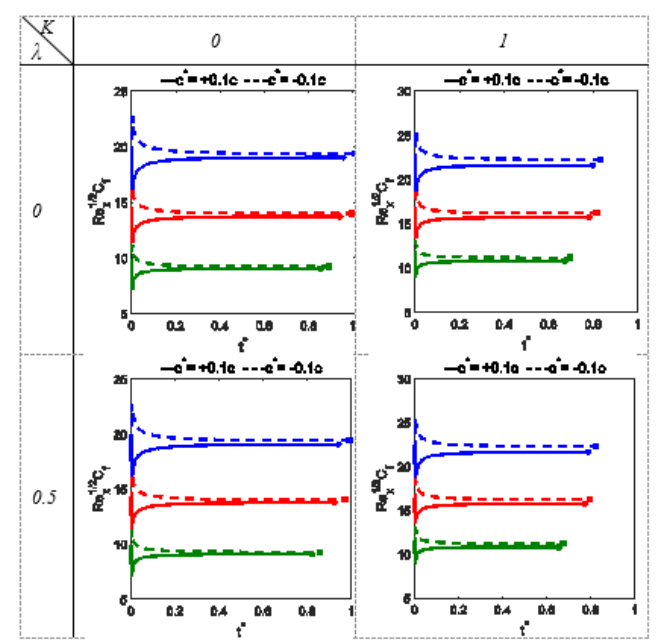

Fig. 7: variation of local Nusselt number of shrinking sheet with time for different Nano particle volume fraction $(\phi=0,0.1,0.2)$, different heat generation parameter and different porous parameter for cu-water nanofluid.

$\left(\mathrm{Pr}_{\mathrm{eff}}=3.5 \mathrm{~S}=0.1 \mathrm{a} / \mathrm{c}=2 \Lambda=0.5\right)$

\section{Conclusions}

In this paper we discussed unsteady two dimensional stagnation point flow of a $\mathrm{cu}-$ water nanofluid for stretching and shrinking sheet in detail. The initial steady state was considered perturbed by a step change in the stretching/shrinking sheet velocity that affects both flow velocity and temperature distribution. Effects of heat generation, volume fraction and porosity on convergence time and heat transfer parameters has been investigated and variations of Nusselt number and skin friction coefficient with time has been analyzed. Stretching sheet shows higher values in Nusselt number while shrinking sheet represents higher values of skin friction compared to stretching sheet. Results show that by increasing sheet velocity, skin friction increases while Nusselt number decreases for both stretching and shrinking sheets. Also it was shown that the drop in shrinking sheet Nusselt number value is more significant compared to stretching sheet while stretching sheet presents higher variation in skin friction coefficient with time compared to shrinking sheet.

\section{References}

[1] S. U. S. Choi, Z. G. Zhang, W. Yu, F. E. Lockwood, and E. A. Grulke, "Anomalous thermal conductivity enhancement in nanotube suspensions," Appl. Phys. Lett., vol. 79, no. 14, pp. 2252-2254, 2001. 
[2] S. U. S. Choi, "Enhancing Thermal Conductivity of Fluids with Nanoparticles," Proc. 1995 ASME Int. Mech. Engineering Congress Expo. San Francisco, USA, ASME, FED231/MD66, pp. 99-105, 1995.

[3] R. Saidur, K. Y. Leong, and H. a. Mohammad, "A review on applications and challenges of nanofluids," Renew. Sustain. Energy Rev., vol. 15, no. 3, pp. 1646-1668, 2011.

[4] V. Trisaksri and S. Wongwises, "Critical review of heat transfer characteristics of nanofluids," Renew. Sustain. Energy Rev., vol. 11, no. 3, pp. 512-523, 2007.

[5] H. Masuda, A. Ebata, and K. Teramae, "Alteration of thermal conductivity and viscosity of liquid by dispersing ultra-fine particles. Dispersion of $\mathrm{Al} 2 \mathrm{O} 3, \mathrm{SiO} 2$ and $\mathrm{TiO} 2$ ultra-fine particles," Thermophysical properties, vol. 7, no. 4, pp. 227-233, 1993.

[6] Y. J. Hwang, Y. C. Ahn, H. S. Shin, C. G. Lee, G. T. Kim, H. S. Park, and J. K. Lee, "Investigation on characteristics of thermal conductivity enhancement of nanofluids," Curr. Appl. Phys., vol. 6, no. 6 SPEC. ISS. pp. 1068-1071, 2006

[7] D.-H. Yoo, K. S. Hong, and H.-S. Yang, "Study of thermal conductivity of nanofluids for the application of heat transfer fluids," Thermochim. Acta, vol. 455, no. 1-2, pp. 66-69, 2007.

[8] S. W. W. Daungthongsuk, "A critical review of convective heat transfer of nanofluids," Renew. Sustain. Energy Rev., pp. 1-23, 2005.

[9] S. Kakaç and A. Pramuanjaroenkij, "Review of convective heat transfer enhancement with nanofluids," Int. J. Heat Mass Transf., vol. 52, no. 13-14, pp. 3187-3196, 2009.

[10] D. Wen and Y. Ding, "Experimental investigation into convective heat transfer of nanofluids at the entrance region under laminar flow conditions," Int. J. Heat Mass Transf., vol. 47, no. 24, pp. 5181-5188, 2004.

[11] K. H. Solangi, S. N. Kazi, M. R. Luhur, A. Badarudin, A. Amiri, R. Sadri, M. N. M. Zubir, S. Gharehkhani, and K. H. Teng, "A comprehensive review of thermo-physical properties and convective heat transfer to nanofluids," Energy, vol. 89, pp. 1065-1086, 2015.

[12] D. Wen and Y. Ding, "Formulation of nanofluids for natural convective heat transfer applications," International Journal of Heat and Fluid Flow, vol. 26, no. 6, pp. 855-864, 2005.

[13] K. N. Lakshmisha, S. Venkateswaran, and G. Nath, "Three-dimensional unsteady flow with heat and mass transfer over a continuous stretching surface," ASME J. Heat Transf., vol. 110, no. August 1988, pp. 590-595, 1988.

[14] N. Bachok, A. Ishak, and I. Pop, "Unsteady boundary-layer flow and heat transfer of a nanofluid over a permeable stretching / shrinking sheet," International Journal of Heat and Mass Transfer, vol. 55, no. 7-8, pp. 2102-2109, 2012.

[15] N. Bachok, A. Ishak, and I. Pop, "Flow and heat transfer over a rotating porous disk in a nanofluid," Phys. $B$ Condens. Matter, vol. 406, no. 9, pp. 1767-1772, 2011.

[16] E. M. A. Elbashbeshy, "Heat transfer in a porous medium over a stretching surface with internal heat generation and suction or injection," Applied Mathematics and Computation, vol. 158, no. 3, pp. 799-807, 2004.

[17] R. Nazar, N. Amin, D. Filip, and I. Pop, "Unsteady boundary layer flow in the region of the stagnation point on a stretching sheet," Int. J. Eng. Sci., vol. 42, no. 11-12, pp. 1241-1253, 2004.

[18] M. A. A. Hamad and I. Pop, "Scaling Transformations for Boundary Layer Flow near the Stagnation-Point on a Heated Permeable Stretching Surface in a Porous Medium Saturated with a Nanofluid and Heat Generation/Absorption Effects," Transp. Porous Media, vol. 87, no. 1, pp. 25-39, 2010.

[19] P. Rana and R. Bhargava, "Commun Nonlinear Sci Numer Simulat Flow and heat transfer of a nanofluid over a nonlinearly stretching sheet: A numerical study," Commun. Nonlinear Sci. Numer. Simul., vol. 17, no. 1, pp. 212226, 2012.

[20] D. Pal, G. Mandal, and K. Vajravalu, "Mixed convection stagnation-point flow of nanofluids over a stretching/ shrinking sheet in a porous medium with internal heat generation / absorption," Communications in Numerical Analysis, vol. 2015, no. 1, pp. 30-50, 2015.

[21] E. M. A. Elbashbeshy, D. A. Aldawody, "Effects of Thermal Radiation and Magnetic Field on Unsteady Mixed Convection Flow and Heat Transfer Over a Porous Stretching Surface Equations of motion," International Journal of Nonlinear Science, vol. 9, no. 4, pp. 448-454, 2010.

[22] R. A. Van Gorder, E. Sweet, and K. Vajravelu, "Nano boundary layers over stretching surfaces," Commun. Nonlinear Sci. Numer. Simul., vol. 15, no. 6, pp. 1494-1500, 2010. 